\title{
Cannabidiol Does Not Impair Anabolic Signaling Following Eccentric Contractions in Rats
}

\author{
Henning T. Langer \\ University of California \\ Suraj Pathak \\ University of California
}

\author{
Agata A. Mossakowski \\ University of California and Charité-Universitätsmedizin Berlin
}

\author{
Mark Mascal \\ University of California Davis
}

\author{
Keith Baar \\ University of California and VA Northern California Health Care System
}

\begin{abstract}
Cannabidiol (CBD) has proven clinical benefits in the treatment of seizures, inflammation, and pain. The recent legalization of $\mathrm{CBD}$ in many countries has caused increased interest in the drug as an over-the-counter treatment for athletes looking to improve recovery. However, no data on the effects of $\mathrm{CBD}$ on the adaptive response to exercise in muscle are available. To address this gap, we eccentrically loaded the tibialis anterior muscle of 14 rats, injected them with a vehicle $(n=7) \mathrm{or} 100 \mathrm{mg} / \mathrm{kg} \mathrm{CBD}(n=7)$, and measured markers of injury, inflammation, anabolic signaling, and autophagy $18 \mathrm{hr}$ later. Pro-inflammatory signaling through nuclear factor kappa B (NF-kB) (Ser536) increased with loading in both groups; however, the effect was significantly greater $(36 \%)$ in the vehicle group $(p<.05)$. Simultaneously, anabolic signaling through ribosomal protein S6 kinase beta-1 (S6K1) (Thr389) increased after eccentric contractions in both groups with no difference between vehicle and CBD $(p=.66)$. The ribosomal protein S6 phosphorylation $(240 / 244)$ increased with stimulation $(p<.001)$ and tended to be higher in the CBD group $(p=.09)$. The ubiquitin-binding protein p62 levels were not modulated by stimulation $(p=.6)$, but they were $46 \%$ greater in the CBD compared with the vehicle group $(p=.01)$. Although liver weight did not differ between the groups $(p=.99)$ and levels of proteins associated with stress were similar, we did observe serious side effects in one animal. In conclusion, an acute dose of CBD decreased pro-inflammatory signaling in the tibialis anterior without blunting the anabolic response to exercise in rats. Future research should determine whether these effects translate to improved recovery without altering adaptation in humans.
\end{abstract}

Keywords: CBD, inflammation, muscle, mTORC1, recovery

Cannabidiol (CBD), a major chemical constituent of the Cannabis sativa plant, is one of over 100 documented cannabinoids and has been associated with analgesic, anti-inflammatory, neuroprotective, and antioxidant properties (Couch, Maudslay, Doleman, Lund, \& O’Sullivan, 2018; Iskedjian, Bereza, Gordon, Piwko, \& Einarson, 2007; Lattanzi et al., 2018). Recently, two CBD-containing drugs have been approved by the U.S. Food and Drug Administration as well as the European Union to treat diseases such as epilepsy and symptoms of multiple sclerosis (U.S. Food and Drug Administration, 2020). Unlike the other major cannabinoid found in $C$. sativa, tetrahydrocannabinol (THC), CBD has no psychotropic action and is becoming prevalent as an over-the-counter medication in a growing number of countries. Hemp is a $C$. sativa, variety characterized by a low THC content, whereas the percentage of THC can be $>20 \%$ in other cultivars

Langer and Baar are with the Department of Physiology and Membrane Biology, University of California, Davis, Davis, CA, USA. Mossakowski is with the Charité-Universitätsmedizin Berlin, corporate member of Freie Universität Berlin, Humboldt-Universität zu Berlin, and Berlin Institute of Health, Berlin, Germany. Mossakowski, Pathak, and Baar are with the Department of Neurobiology, Physiology and Behavior, University of California, Davis, Davis, CA, USA. Mascal is with the Department of Chemistry, University of California, Davis, Davis, CA, USA. Baar is also with the VA Northern California Health Care System, Mather, CA, USA. Langer (htlanger@ucdavis.edu) is corresponding author. generally characterized as marijuana. As part of the Farm Bill, signed in December 2018, the United States has federally legalized the growing of hemp that contains less than $0.3 \%$ THC (dry weight; U.S. Food and Drug Administration, 2020). As such, CBD has enjoyed a tremendous rise in popularity among chronically ill patients.

Another population that is increasingly turning to $\mathrm{CBD}$ is athletes looking for recovery aids that allow a greater training load. In contact sports such as rugby, as many as $26 \%$ of players self-reported current or past use of CBD with the number reaching approximately $40 \%$ in the oldest age group, that is, between 28 and 33 years (Kasper et al., 2020). The primary reasons reported for taking CBD were relief of pain and improved recovery. These data are consistent with another study that found that approximately $61 \%$ of athletes who currently use cannabis (i.e., CBD, THC, or both) do so to alleviate pain (Zeiger, Silvers, Fleegler, \& Zeiger, 2019).

Although viewed as relatively safe, CBD is not risk-free and has shown dose-dependent adverse effects in humans, ranging from loss of appetite and somnolence to liver abnormalities (Huestis et al., 2019). The analgesic and anti-inflammatory effects of CBD desired by athletes are well documented for certain patient groups, but there is a paucity of information on the benefits and potential side effects of CBD in the context of exercise. For other drugs that are used to enhance recovery from exercise-induced muscle damage (EIMD), such as nonsteroidal anti-inflammatory 
drugs (NSAIDs), documented adverse effects include bleeding, cardiovascular complications, and renal damage (Pirmohamed et al., 2004). Additionally, there is evidence that the improved recovery may be associated with impaired adaptations to training. For example, NSAID use blunts satellite cell activation following exercise (Bondesen, Mills, Kegley, \& Pavlath, 2004; Mackey et al., 2007) and impairs protein metabolism and muscle growth (Rodemann \& Goldberg, 1982; Soltow et al., 2006). Although the data in humans are more equivocal, some studies have found that NSAIDs hamper mixed muscle protein and collagen synthesis (Dideriksen, 2014; Trappe et al., 2002). Other common self-medications, such as alcohol, have been shown to reduce the activation of the mechanistic target of rapamycin complex 1 (mTORC1) in model organisms (Lang et al., 2003) and myofibrillar protein synthesis in humans following resistance-type exercise (Parr et al., 2014).

To determine whether acute treatment with CBD decreases inflammation and anabolic signaling following eccentric contractions, we measured the phosphorylation of mTORC1 targets, inflammation, and levels of autophagy-related markers and force transfer proteins $18 \mathrm{hr}$ after eccentric loading in rats. We hypothesized that CBD would decrease inflammatory markers and blunt the anabolic response to exercise.

\section{Methods}

\section{Animals}

All experiments were approved and carried out according to a protocol approved by the Institutional Animal Care and Use Committee of the University of California Davis. Fourteen (vehicle: $n=7$; CBD: $n=7$ ) female Sprague Dawley rats (mean $\pm S D=$ $253 \pm 21 \mathrm{~g}$ ) were selected to study the effects of CBD after acute eccentric loading. Animals were housed in 12:12 hr light-dark cycles and allowed to feed ad libitum.

\section{Electrostimulation and Drug Administration}

Stimulation of the sciatic nerve causing eccentric contractions of the tibialis anterior (TA) muscle was carried out as described previously (West et al., 2016). Each contraction was induced by a 4-6 V stimulus for $2 \mathrm{~s}$, followed by a relaxation period of $8 \mathrm{~s}$. Six contractions composed one set, after which the animal was rested for $1 \mathrm{~min}$. After 10 sets, the stimulation protocol was concluded and each animal was injected with either vehicle (Ethanol + Tween-20+Saline $[0.9 \%$ $\mathrm{NaCl}], 1: 1: 8$ ) or $100 \mathrm{mg} / \mathrm{kg}$ CBD dissolved in the vehicle. The CBD product (Amber Metric, Santa Rosa, CA) was laboratory tested and hemp derived (99.8\% purity). Muscles and liver were collected $18 \mathrm{hr}$ after the stimulation protocol ended (Figure 1a). The muscles from both hindlimbs were removed, rinsed in phosphate-buffered saline to remove blood, blotted dry, and weighed. The TA muscle was pinned on cork at resting length and frozen in liquid nitrogen-cooled isopentane for histological and biochemical analyses.

\section{Histochemistry}

Serial cross-sections $(10 \mu \mathrm{m})$ were cut from the TA muscle using a cryostat (Leica CM3050 S; Leica Microsystems, Buffalo Grove, IL). For the hematoxylin and eosin staining, sections were brought to room temperature, then submerged in $90 \%$ ethanol alcohol, followed by tap water for $30 \mathrm{~s}$. On each slide, $400 \mu \mathrm{l}$ Mayer's hemalum solution (Merck Group, Darmstadt, Germany) was applied, and slides were incubated for $6 \mathrm{~min}$. Slides were blued under running tap water for 2 min, then dipped in tap water for another $8 \mathrm{~min}$. Then, $300 \mu \mathrm{l} 0.5 \%$ aqueous eosin $\gamma$-solution (Merck Group) was applied to each slide for 2 min. Sections were dehydrated in $90 \%$ and then $100 \%$ ethanol alcohol for 1 min each. The alcohol was removed by dipping sections in xylene for $5 \mathrm{~min}$. After sections were air dried, they were mounted with DPX Mountant (Sigma-Aldrich, St Louis, MO) to prepare them for imaging.

\section{Western Blotting}

The distal section of the TA muscle and the liver were separately powdered and homogenized in $200 \mu \mathrm{l}$ sucrose lysis buffer, vortexed, and normalized for protein content as described previously (West et al., 2016). A total of 30-60 $\mu \mathrm{g}$ of protein were loaded per lane, run for $40 \mathrm{~min}$ at $200 \mathrm{~V}$, and transferred onto polyvinylidene fluoride or nitrocellulose membrane in an ice-cold transfer buffer at $100 \mathrm{~V}$ for $30 \mathrm{~min}$. Membranes were blocked in 1\% fish skin gelatin dissolved in Tris-buffered saline with $0.1 \%$ Tween-20 for $30 \mathrm{~min}$ and probed with the primary antibody overnight. The following antibodies were used. Cell Signaling (Cell Signaling Technology, Danvers, MA): S6K1 (Thr389) (\#9205; lot 16), ribosomal protein S6 (Ser240/244) (\#5364), eukaryotic translation initiation factor 4E-binding protein 1 (4E-BP1) (Thr37/46) (\#2855), microtubuleassociated proteins 1A/1B light chain 3B (LC3B) (\#2775; lot 10), heat shock protein beta-1 (HSP27) (\#2402; lot 8), heat shock protein HSP 90-alpha (HSP90) (\#4877; lot 5), unc-51 like autophagy activating kinase 1 (ULK1) (Ser757) (\#14202; lot 1), p62 (\#5114; lot 4), NF-kB (Ser536) (\#3033; lot14), Annexin A2 (\#8235; lot 2), C/EBP-homologous protein (CHOP) (\#5554); Santa Cruz (Santa Cruz Biotechnology Inc., Dallas, TX): Dystrophin (\#365954; lot E2711), Dysferlin (\#16635; lot H162), Desmin (\#271677; lot F1913), muscle LIM protein (mLIM)/cysteine and glycine-rich protein 3 (mLIM) (\#166930; lot E2814); Millipore Sigma (Merck Group): insulin receptor substrate 1 (IRS1) (\#06248; lot 2465193); Enzo (Enzo Life Sciences Inc., Farmingdale, NY): $\alpha$ B Crystallin (ADI-SPA-223; lot 05011812). Levels of every protein were normalized to total protein content per lane as assessed via Ponceau $\mathrm{S}$ staining of the membrane or a fluorescent gel (Gilda \& Gomes, 2013).

\section{Serum Creatine Kinase Levels}

Serum creatine kinase activity was measured using a Creatine Kinase Activity Assay Kit (Sigma-Aldrich, St. Louis, MO) according to the manufacturer's protocol.

\section{Statistics}

Data analysis was carried out using Graphpad Prim 8.0.0 (GraphPad Software, San Diego, CA). Paired $t$ tests or two-way analysis of variance (Stimulation and CBD) combined with Tukey's multiple comparison test were applied depending on the number of groups and variables. The alpha level was set at $p=.05 ; p$ values $<.05$ were deemed statistically significant, and $p$ values between .05 and .1 were described as trends.

\section{Results}

\section{Electrostimulation Transiently Increases Muscle Weight in Rats}

Ten sets of six eccentric contractions at $100 \mathrm{~Hz}$ caused a mean increase of $6 \%$ muscle mass $(31 \pm 23 \mathrm{~g})$ in 13 out of 14 TA muscles 
(a) Time
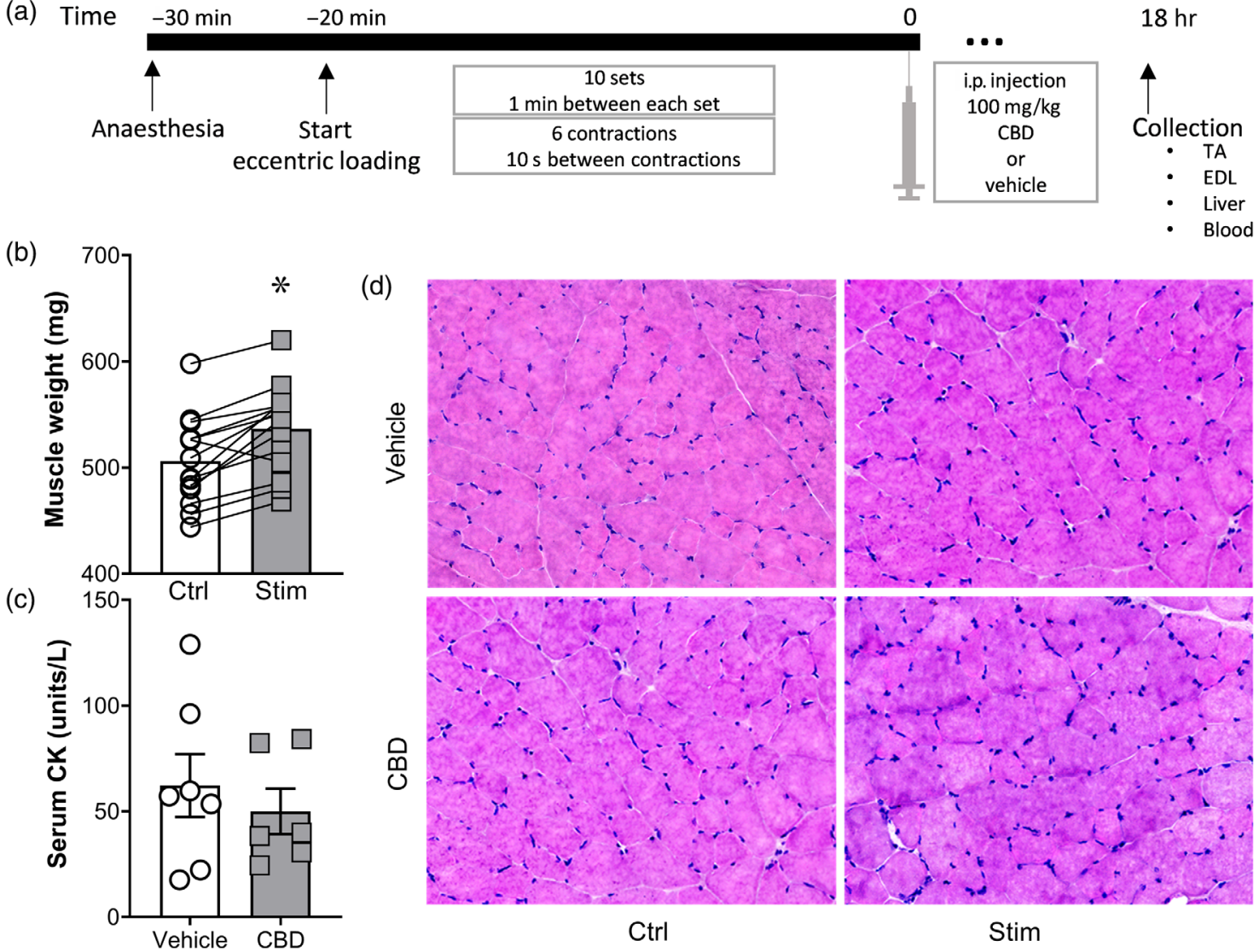

Ctrl

Stim

Figure 1 - Experimental design, changes in muscle weight, serum CK, and histology. (a) Timeline of the experiment. Fourteen animals $(n=7$ vehicle; $n=7 \mathrm{CBD}$ ) were anesthetized and the sciatic nerve stimulated to cause eccentric contractions of the TA. Following 10 sets of six contractions, animals were injected i.p. either with vehicle (Ethanol + Tween-20 $\mathrm{NaCl}, 1: 1: 8)$ or $100 \mathrm{mg} / \mathrm{kg} \mathrm{CBD}$. Eighteen hours later, the muscle tissue was collected. (b) Changes in muscle weight $18 \mathrm{hr}$ after eccentric contractions. Muscle weight increased in 13 out of 14 animals $(p<.001)$. (c) Serum CK levels did not differ between the vehicle or the CBD group at the point of collection. (d) H\&E staining of control and stimulated muscle of vehicle- and CBD-treated animals. No histopathological signs for any of the conditions were observed. Ctrl $=$ control; Stim $=$ stimulated; i.p. $=$ intraperitoneal; EDL $=$ extensor digitorum longus; $\mathrm{CBD}=$ Cannabidiol; $\mathrm{CK}=$ creatine kinase; $\mathrm{NaCl}=$ sodium chloride; $\mathrm{TA}=$ tibialis anterior. ${ }^{*}$ Significant $p$ value is at least $<.05$.

when collected $18 \mathrm{hr}$ later $(p<.001$; Figure $1 \mathrm{~b})$. This transient increase in muscle mass is most likely a physiological function of increased blood flow to the region as well as fluid accumulation resulting from EIMD. No histological signs of cellular damage such as central nuclei, necrosis, or nerve damage were observed via hematoxylin and eosin staining in the control or stimulated TA sections of the vehicle and CBD group (Figure 1d). Serum creatine kinase levels were similar for the vehicle and $\operatorname{CBD}(p=.5$; Figure 1c).

\section{mTORC1 Signaling and Protein Degradation}

Proteins upstream of mTORC1, such as IRS1, did not differ between the vehicle and the CBD group ( $p=.7$; Figure $2 \mathrm{a})$. The stimulation caused a robust increase in S6K1 (Thr389) levels $(p<.001$; Figure 2b). The effects of stimulation on S6K1 phosphorylation were similar for both groups $(p=.5)$. Levels of ribosomal protein S6, phosphorylated at Ser240/244, the downstream substrate of S6K1 were also increased through stimulation $(p<.001)$, and there was a trend for it to be systemically elevated with CBD ( $p=.09$; Figure 2c). Another downstream target of mTORC1, 4E-BP1 (Thr37/46), was not changed with either stimulation $(p=.16)$ or $\mathrm{CBD}(p=.4$; Figure $2 \mathrm{~d})$. The phosphorylation of the autophagy regulator ULK-1 tended to increase with stimulation $(p=.094)$, and this was more pronounced in the CBD group $(p=.2$ for a drug effect, $p=.17$ for an interaction effect; Figure 2e). To estimate autophagic activity, we measured the content of p62 and the whole and cleaved LC3B protein. Although p62 levels were not impacted by stimulation $(p=.6)$, they were increased with CBD ( $p=.01$; Figure $2 \mathrm{f}$ ). On average, p62 levels were $46 \%$ higher in the CBD group compared with the vehicle group (combined effect on control and stimulation). Stimulation decreased the ratio of LC3 II to LC3 I in both groups $(p=.01$; Figure $2 \mathrm{~g}$ ) with the decrease being $21 \%$ less in the CBD group without reaching statistical significance $(p=.17)$.

\section{Structural Proteins, Aggregates, and Inflammation}

Neither Dystrophin nor Desmin levels were affected by either stimulation ( $p=.4$ and $p=.19$, respectively) or CBD $(p=.5$ and $p=.14$, respectively; Figure $3 \mathrm{a}$ and $3 \mathrm{~b}$ ). Dysferlin, a protein important for membrane repair, as well as its close partner Annexin A2 were also unchanged with stimulation $(p=.8$ and $p=.9$, respectively) and CBD ( $p=.6$ and 0.9 ; Figure $3 c$ and $3 d)$. The structural/signaling protein $\mathrm{mLIM}$ increased with both stimulation 


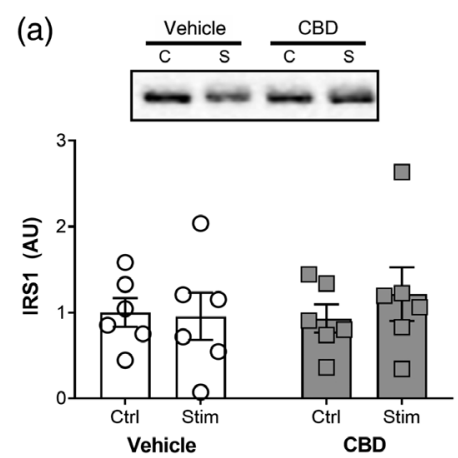

(e)
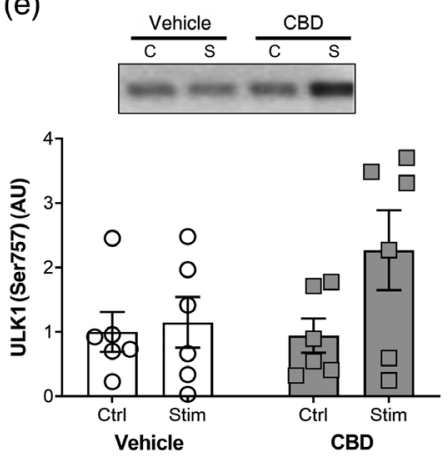

(b)

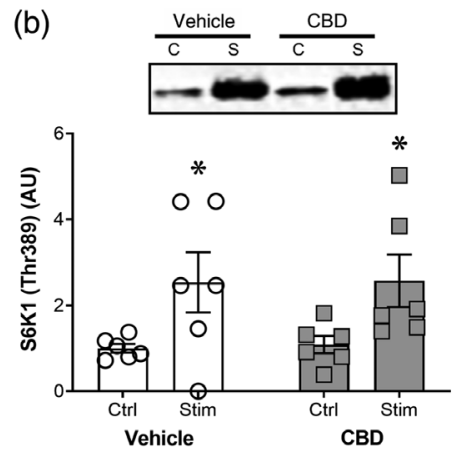

(f)

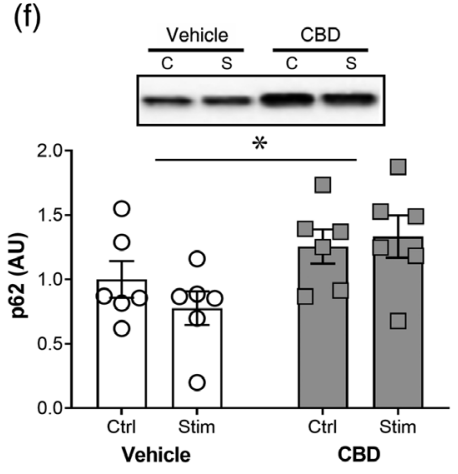

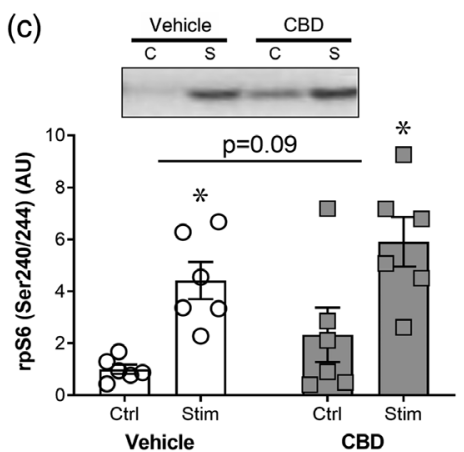

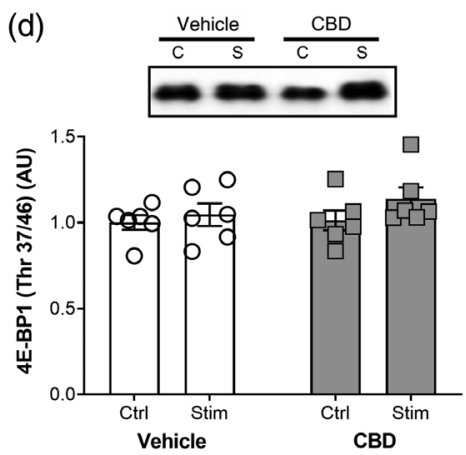

(g)
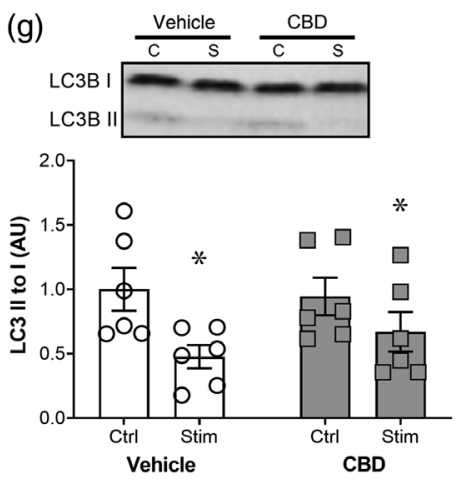

(h)
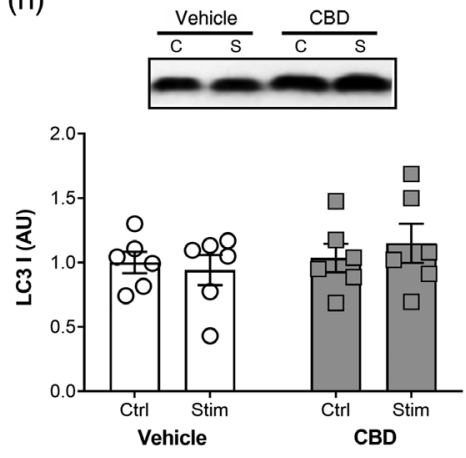

Figure 2 - Immunoblots of mTORC1 signaling and autophagy after eccentric contractions with CBD. Levels of (a) IRS1, (b) S6K1 (Thr389), (c) rpS6 (Ser240/244), (d) 4E-BP1 (Thr37/46), (e) ULK1 (Ser757, (f) p62, (g) LC3 II to I ratio, and (h) LC3 I are shown 18 hr following eccentric contractions and treatment with either vehicle or CBD. All graphs show protein levels relative to total protein content of their respective lane on the gel or membrane. $\mathrm{Ctrl}=$ control; $\mathrm{Stim}=$ stimulated $; \mathrm{CBD}=$ Cannabidiol; $\mathrm{mTORC} 1=$ mechanistic target of rapamycin complex 1 ; rpS6 = ribosomal protein S6. * Significant $p$ value of at least $<.05$.

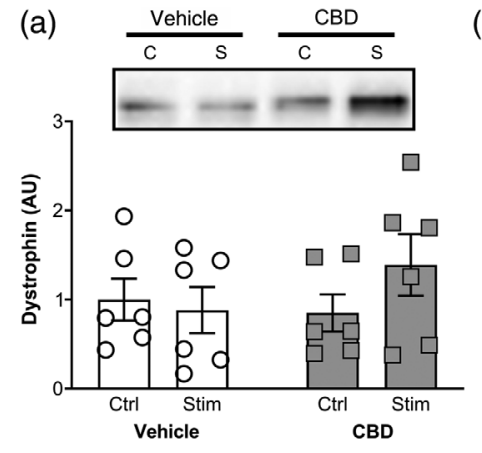

(e)
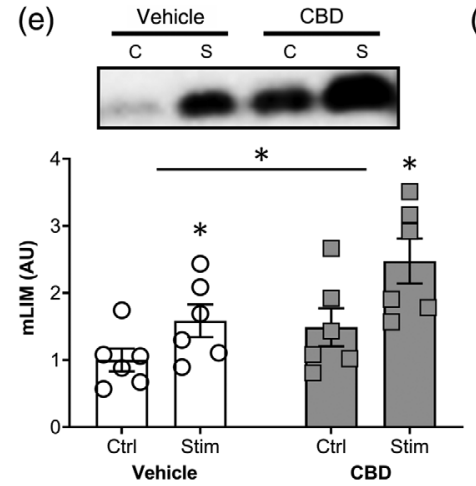

(b)

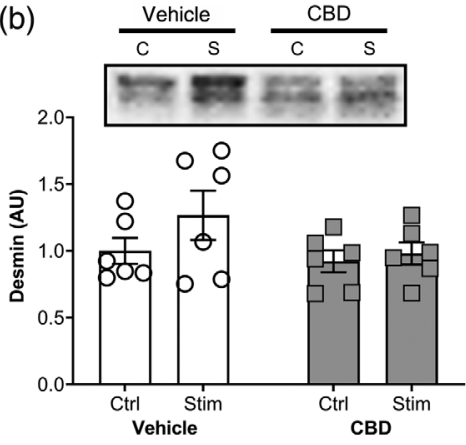

(f)
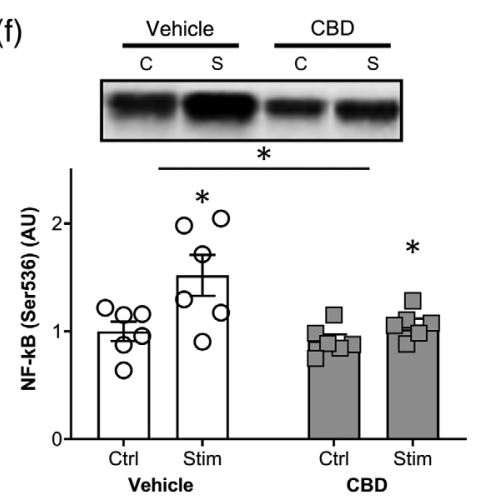

(c)

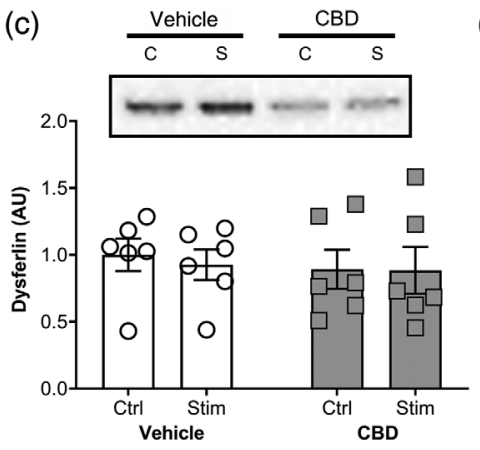

(g)
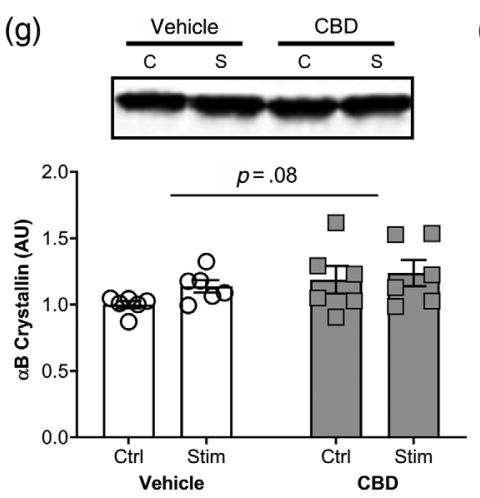

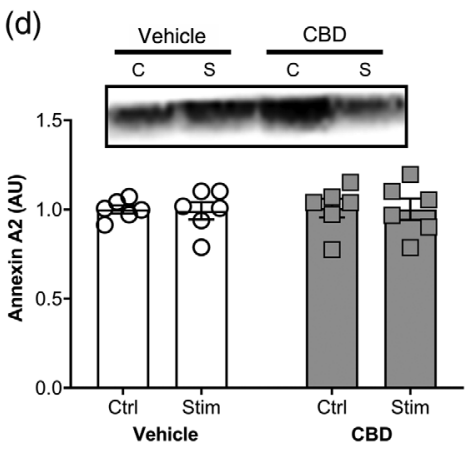

(h)
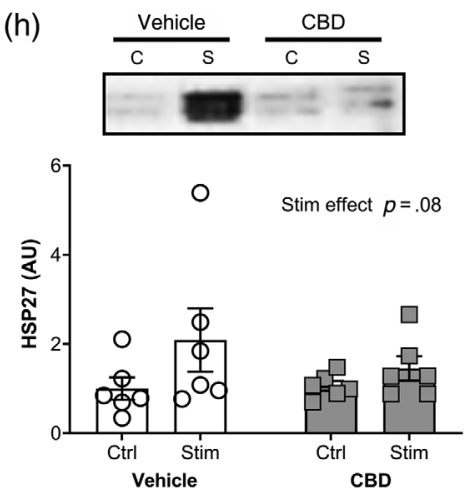

Figure 3 - Structural proteins, inflammatory signaling, and sensors of cellular stress in muscle after eccentric contractions and CBD treatment. Levels of (a) Dystrophin, (b) Desmin, (c) Dysferlin, (d) Annexin A2, (e) mLIM, (f) phosphorylated NF-kB (Ser536), (g) $\alpha$ B Crystallin, and (h) HSP27 levels are shown $18 \mathrm{hr}$ following eccentric contractions and treatment with either vehicle or CBD. All graphs show protein levels relative to total protein content of their respective lane on the gel or membrane. $\mathrm{Ctrl}=$ control; $\mathrm{Stim}=$ stimulated; $\mathrm{CBD}=\mathrm{Cannabidiol.}{ }^{*}$ Significant $p$ value of at least $<.05$. 
$(p=.01)$ and CBD $(p=.02)$. Mean mLIM levels in the CBD group were $53 \%$ higher than in the vehicle group (control and stimulation combined; Figure 3e). Phosphorylation of the proinflammatory transcription factor NF-kB (Ser536) was increased with stimulation $(p<.01$; Figure $3 \mathrm{f})$ with the increase for CBD $(17 \pm 11 \%)$ being less than for the vehicle group (53 $\pm 36 \%$; $p<.05)$. The $\alpha \mathrm{B}$ crystallin, a molecular chaperone, tended to show a drug effect $(p=.08)$, which was reflected in the vehicle group, starting lower and increasing with stimulation. Baseline levels of $\alpha \mathrm{B}$ crystallin tended to increase with CBD (Figure 3g). The HSP27 tended to increase with stimulation $(p=.08)$. The increase in HSP27 with eccentric loading in the vehicle group $(130 \pm 76 \%)$ tended to be greater than that for the CBD group $(36 \pm 43 \% ; p=.09 ;$ Figure $3 \mathrm{~h})$.

\section{Liver Metabolism, Inflammation, and Toxicity}

Liver weights were identical between groups ( $p=.99$; Figure $4 \mathrm{a})$. Markers of stress measured were similar between the vehicle and the CBD groups. An increase in LC3 I levels was observed with CBD $(p=.01$; Figure $4 \mathrm{f})$. This led to a mildly decreased $(24 \%)$ LC3 II to LC3 I ratio in the CBD compared with the vehicle group $(p=.19)$, suggesting decreased autophagy (Figure 4e).

\section{Discussion}

The goal of this study was to investigate the effects of a single dose of CBD on acute postexercise signaling in skeletal muscle. We stimulated the sciatic nerve of rats, causing strong, eccentric (a)

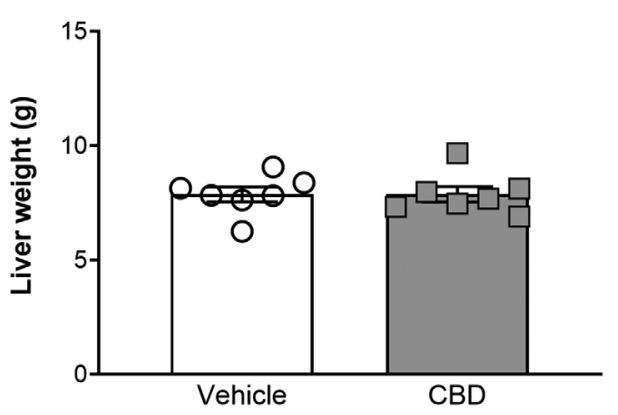

(d)
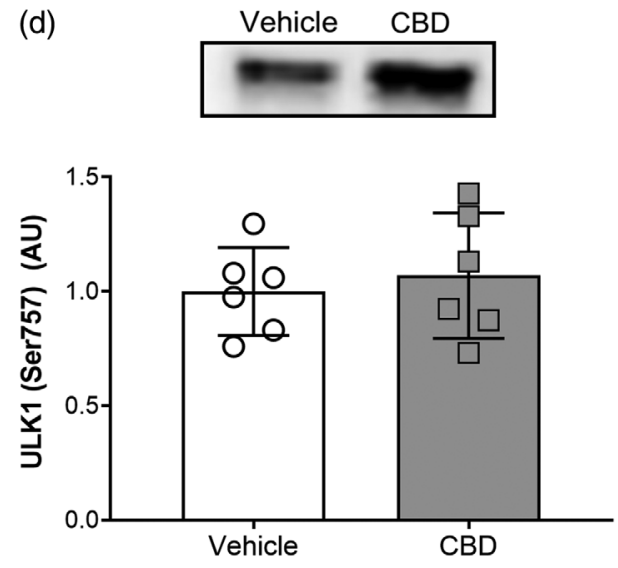

(g)

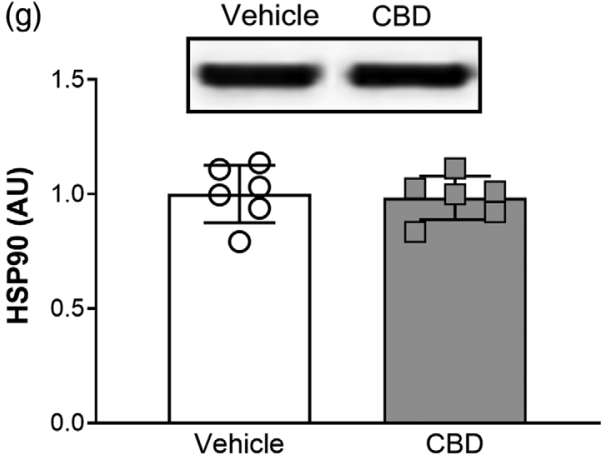

(b)

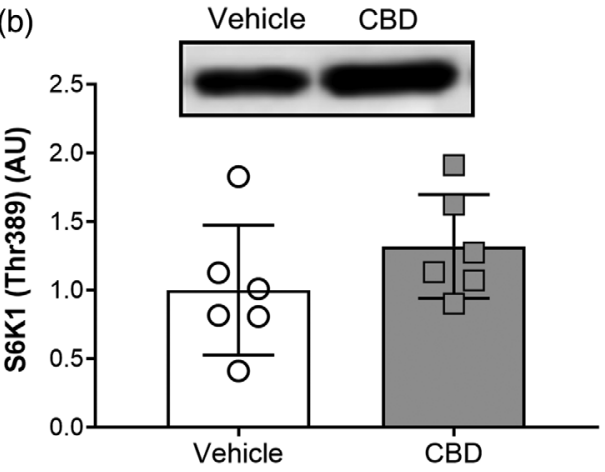

(e)

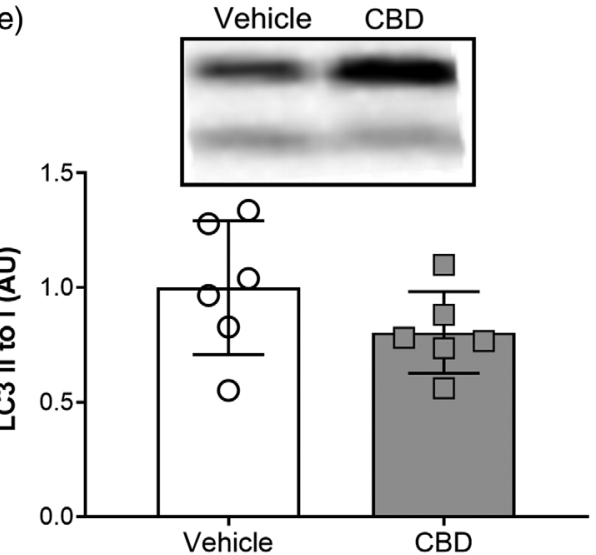

(h)

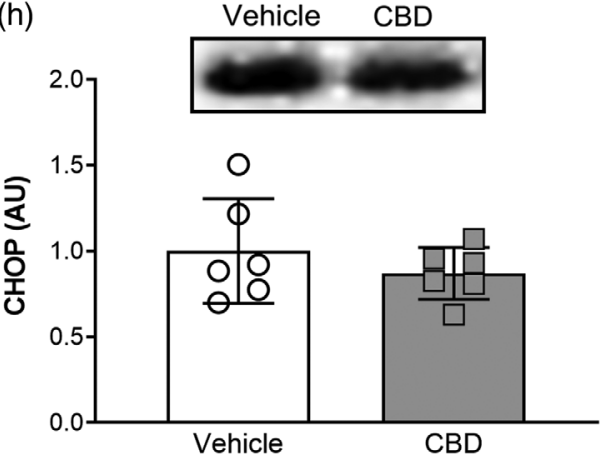

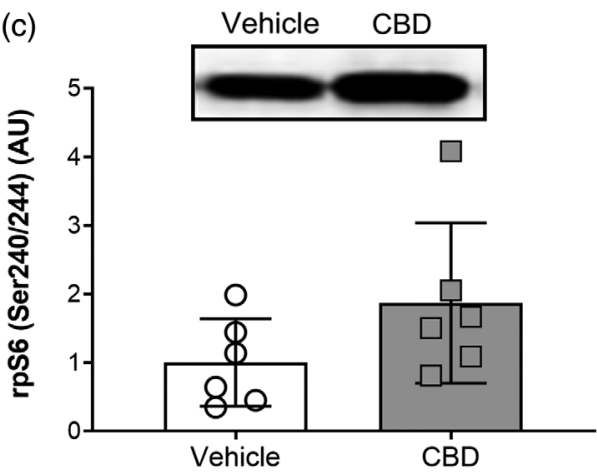

(f)
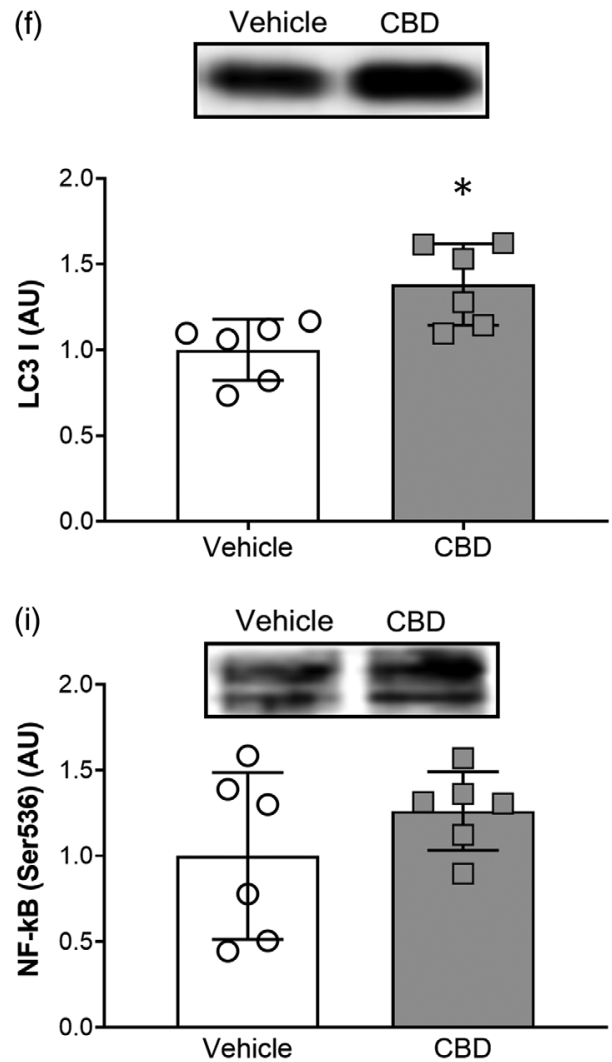

Figure 4 - Liver toxicity and protein levels related to stress, inflammation, and tissue growth. The (a) liver weights and the levels of (b) phosphorylated S6K1 (Thr389), (c) rpS6 (Ser240/244), (d) phosphorylated ULK1 (Ser757), (e) LC3 II to I ratio, (f) LC3 I protein, (g) HSP90, (h) CHOP, and (i) phosphorylated NF-kB (Ser536) are shown $18 \mathrm{hr}$ following eccentric contractions and treatment with either vehicle or CBD. All graphs show protein levels relative to total protein content of their respective lane on the gel or membrane. $\mathrm{CBD}=\mathrm{Cannabidiol}$; rpS6 $=$ ribosomal protein $\mathrm{S} 6$.

*Significant $p$ value of at least <.05. 
contractions of the TA muscle that are known to increase anabolic signaling and protein synthesis for more than $24 \mathrm{hr}$ (Baar \& Esser, 1999). Immediately following a total of 60 contractions, we injected the animals with either a vehicle or a $100 \mathrm{mg} / \mathrm{kg}$ of CBD (Figure 1a) and collected muscle for analysis $18 \mathrm{hr}$ later. We found that the overall effects of $\mathrm{CBD}$ on the response to exercise were relatively mild. Most importantly, we found that despite decreasing pro-inflammatory signaling, CBD did not affect anabolic signaling through the mTORC1 axis (Figure 2b-2d).

Based on its documented medical properties, the fact that it was recently legalized in many countries and removed from the list of performance-enhancing drugs prohibited by the World AntiDoping Association, CBD is becoming increasingly popular as an over-the-counter drug for the general population and athletes. The latter seek relief of chronic pain, EIMD, or improved recovery following intense training and competition (Kasper et al., 2020; Zeiger et al., 2019). However, much of the evidence that promotes the use of CBD by athletes is anecdotal. There is a scarcity of data describing the effects of CBD in skeletal muscle and adaptation to exercise. Other over-the-counter or recreational drugs such as NSAIDs and alcohol can decrease inflammation, but this can come at the expense of decreased satellite cell proliferation as well as anabolic signaling and muscle and collagen synthesis (Bondesen et al., 2004; Dideriksen, 2014; Mackey et al., 2007; Rodemann \& Goldberg, 1982; Soltow et al., 2006; Trappe et al., 2002). These actions suggest that prolonged NSAID use may hamper adaptation to exercise and muscle growth (Schoenfeld, 2012). Interestingly, our data indicate that although an acute dose of CBD has antiinflammatory effects on skeletal muscle (blunted NF-kB phosphorylation in response to training; Figure 3f), it does not negatively affect anabolic signaling through the mTORC1 pathway (Figure $2 b-$ $2 \mathrm{~d})$. If anything, mTORC1 signaling in muscle and liver tended to be slightly greater in the CBD group (Figures $2 c, 4 b$, and $4 c$ ). The activation of mTORC1 is well known for its role in muscle growth (Baar \& Esser, 1999); however, in the context of EIMD, the more important role for mTORC1 is muscle remodeling (Ge et al., 2009). Therefore, our data suggest that unlike other recreational drugs such as alcohol, CBD does not blunt mTORC1 signaling and may, in fact, provide analgesic and anti-inflammatory signals without limiting recovery.

In $\mathrm{C} 2 \mathrm{C} 12$ cells, primary myoblasts, and satellite cells, CBD can positively affect cell proliferation and myotube formation (Iannotti et al., 2019). The improved proliferation of muscle precursor cells was hypothesized to result primarily from improved autophagy. Consistent with these data, we found indicators of a potential role of CBD in increasing autophagy in muscle. The p62 regulates the formation of autophagosomes and was elevated in CBD muscle compared with the vehicle at $18 \mathrm{hr}$ after eccentric contractions (Figure 2f). The abundance of p62 alone is notoriously difficult to interpret with respect to its role in autophagy (Gottlieb, Andres, Sin, \& Taylor, 2015; Mizushima \& Yoshimori, 2007). However, in concert with monitoring other intracellular protein levels relevant for autophagy, modest conclusions can be drawn (Gottlieb et al., 2015). Specifically, increased p62 levels in the face of ULK1 tending to be higher in the CBD group after stimulation, and a tendency for a smaller decrease in the LC3 II to LC3 I ratio in CBD-treated muscle after exercise, support a potential role for CBD in increasing autophagy. Furthermore, we found mLIM, a structural protein that has an important role in autophagy, to be upregulated through CBD, irrespective of exercise. Together, these data suggest that following eccentric loading in rats, CBD tends to increase autophagy without impacting mTORC1 signaling.
A major concern with any drug is potential toxicity. As CBD is primarily metabolized in the liver (Jiang, Yamaori, Takeda, Yamamoto, \& Watanabe, 2011), we measured liver mass and the levels of stress proteins in the liver. We could not find any strong indicators of cellular stress or apoptosis in the liver of CBD animals compared with vehicle-treated animals. We did, however, note that some of the effects observed for muscle, specifically proinflammatory markers, tended to shift in the opposite manner in the liver (Figure 4). For instance, phosphorylation of NF-kB (Ser536) was lower in the stimulated muscle treated with CBD (Figure 3f), whereas in the liver, this modification tended to increase with CBD (Figure 4i). On the other hand, mTORC1 signaling appeared remarkably similar to what was observed in skeletal muscle with a tendency to be unchanged or mildly increased with CBD compared with the vehicle (Figures $2 b-2 c$ and $4 b-4 c$ ). This suggests that although CBD might share some of its effects across multiple tissues, other tissues might be affected by CBD in a different manner and provides a rationale for further investigation.

\section{Safety Concern}

Despite being well within the documented no-adverse-effect-level for CBD in rats (Center For Drug Evaluation and Research, 2018), we found that one animal treated with CBD exhibited difficulties breathing at the point of collection. Furthermore, all CBD-treated animals showed signs of stomach bloating, particularly in the corpus section. Normally, respiratory and gastrointestinal stresses are not observed with the use of CBD at these dosages in rats (Machado Bergamaschi, Helena Costa Queiroz, Waldo Zuardi, \& Crippa, 2011). However, intravenous experiments in monkeys have shown an LD50 of $200 \mathrm{mg} / \mathrm{kg}$ and difficulties breathing as low as $150 \mathrm{mg} / \mathrm{kg}$ (Huestis et al., 2019; Iffland \& Grotenhermen, 2017). Interspecies differences in physiology and drug metabolism between rats, monkeys, and humans make it challenging to compare results. However, the faster rate of metabolism in rodents explains why most drugs, including CBD, need to be dosed at much higher levels to see effects. For example, a meta-analysis of adjunctive CBD treatment in people suffering from epilepsy found that as little as $10-20 \mathrm{mg} / \mathrm{kg}$ per day had a protective effect against seizures (Lattanzi et al., 2018). However, even at such relatively moderate dosages, $88 \%$ of the patients experienced some sort of adverse effect, and 9\% had to discontinue their CBD treatment based on the adverse effects. Contrast the $20 \mathrm{mg} / \mathrm{kg}$ per day in humans with the rodent models of seizure wherein $200 \mathrm{mg} / \mathrm{kg}$ were administered daily for a prolonged period with no or only minor adverse effects (Iffland \& Grotenhermen, 2017; Patra et al., 2019). These interspecies differences make it impossible to directly extrapolate the results reported here to humans. The key finding of the present study was that the acute effects of CBD on skeletal muscle were mild, even though we injected the rats with a relatively high dose. Whether CBD will have any physiologically meaningful benefits for recreational use in humans, where dosages are much lower and routes of administration are commonly oral or topical, remains unclear.

Future studies will need to investigate the dose-response relationship between the potential benefits of CBD after exercise and potential adverse effects in other tissues, in both acute and chronic settings, in preclinical and clinical studies. The safety profile of CBD should be directly compared with other drugs known for similar effects in respect to inflammation and regeneration to determine the most appropriate solution for athletes. In addition, although CBD is permitted by the World Anti-Doping 
Association, other cannabinoids such as THC are not. For this reason, athletes must pay particular attention to the source and purity of CBD should they decide to use it. Furthermore, it has been proposed that the sedative effects of CBD may involve some in vivo conversion to THC in the acidic gastric tract (Watanabe et al., 2007), although this remains a matter of dispute (Palazzoli et al., 2018).

In summary, this work constitutes the first study on the effects of $\mathrm{CBD}$ on molecular signaling in skeletal muscle following eccentric contractions. We found that an acute dose of CBD did not hamper postexercise anabolic signaling despite decreasing pro-inflammatory signaling and increasing markers of autophagy. Although one animal suffered serious adverse effects from CBD, skeletal muscle and liver showed no signs of tissue toxicity. Even though these data make CBD a potential alternative to common drugs for pain relief and inflammation associated with intense training or contact sports, the possibility remains for serious side effects. This, together with a small body of evidence surrounding the subject of CBD in exercise and sports, should caution athletes against widespread use until more advanced trials in humans have been carried out.

\section{Acknowledgments}

H.T. Langer conducted the animal experiments, tissue collections, skeletal muscle western blots, and creatine kinase assay and drafted the manuscript. A.A. Mossakowski contributed histochemistry, nuclear isolation, and corresponding western blots. S. Pathak was involved with the animal experiments, tissue collections, and liver western blots. M. Mascal and K. Baar helped with the project design and drafting of the manuscript. We would like to thank Sherry Strong, Edward Charles Brummer, and Donald Land for helpful discussions surrounding CBD. We would like to further thank the UC Davis Cannabis and Hemp Research Center for providing the grounds for collaboration and the generous support via the Research Investments in Cannabis and Hemp grant. The research was supported via a Research Investments in Cannabis and Hemp project grant to H.T. Langer. A.A. Mossakowski was supported by a postdoctoral fellowship from Deutsche Forschungsgemeinschaft. The authors have no conflicts of interest.

\section{References}

Baar, K., \& Esser, K. (1999). Phosphorylation of p70S6kcorrelates with increased skeletal muscle mass following resistance exercise. American Journal of Physiology-Cell Physiology, 276, C120-C127.

Bondesen, B. A., Mills, S. T., Kegley, K. M., \& Pavlath, G. K. (2004). The COX-2 pathway is essential during early stages of skeletal muscle regeneration. American Journal of Physiology-Cell Physiology, 287(2), C475-C483. PubMed ID: 15084473 doi:10.1152/ajpcell. 00088.2004

Center For Drug Evaluation and Research, U. F. a. D. A. (2018). Application Number 210365Orig1s000. Retrieved from https:// www.accessdata.fda.gov/drugsatfda_docs/nda/2018/210365Orig1s 000PharmR.pdf

Couch, D. G., Maudslay, H., Doleman, B., Lund, J. N., \& O’Sullivan, S. E. J. I. b. d. (2018). The use of cannabinoids in colitis: A systematic review and meta-analysis. Inflammatory Bowel Diseases, 24(4), 680 697. PubMed ID: 29562280 doi:10.1093/ibd/izy014

Dideriksen, K. (2014). Muscle and tendon connective tissue adaptation to unloading, exercise and NSAID. Connective Tissue Research, 55(2), 61-70. PubMed ID: 24195606 doi:10.3109/03008207.2013.862527
Ge, Y., Wu, A. L., Warnes, C., Liu, J., Zhang, C., Kawasome, H., ... Chen, J. (2009). mTOR regulates skeletal muscle regeneration in vivo through kinase-dependent and kinase-independent mechanisms. American Journal of Physiology-Cell Physiology, 297(6), C1434C1444. PubMed ID: 19794149 doi:10.1152/ajpcell.00248.2009

Gilda, J. E., \& Gomes, A. V. (2013). Stain-free total protein staining is a superior loading control to $\beta$-actin for Western blots. Analytical Biochemistry, 440(2), 186-188. PubMed ID: 23747530 doi:10. 1016/j.ab.2013.05.027

Gottlieb, R. A., Andres, A. M., Sin, J., \& Taylor, D. P. (2015). Untangling autophagy measurements: All fluxed up. Circulation Research, 116(3), 504-514. PubMed ID: 25634973 doi:10.1161/CIRCRESAHA. 116.303787

Huestis, M. A., Solimini, R., Pichini, S., Pacifici, R., Carlier, J., \& Busardò, F. P. J. C. n. (2019). Cannabidiol adverse effects and toxicity. Current Neuropharmacology, 17(10), 974-989. PubMed ID: 31161980 doi:10.2174/1570159X17666190603171901

Iannotti, F. A., Pagano, E., Moriello, A. S., Alvino, F. G., Sorrentino, N. C., D’Orsi, L., .. . De Petrocellis, L. (2019). Effects of non-euphoric plant cannabinoids on muscle quality and performance of dystrophic mdx mice. British Journal of Pharmacology, 176(10), 1568-1584. PubMed ID: 30074247 doi:10.1111/bph.14460

Iffland, K., \& Grotenhermen, F. (2017). An update on safety and side effects of cannabidiol: A review of clinical data and relevant animal studies. Cannabis Cannabinoid Research, 2(1), 139-154. PubMed ID: 28861514 doi:10.1089/can.2016.0034

Iskedjian, M., Bereza, B., Gordon, A., Piwko, C., \& Einarson, T. R. (2007). Meta-analysis of cannabis based treatments for neuropathic and multiple sclerosis-related pain. Current Medical Research Opinion, 23(1), 17-24. PubMed ID: 17257464 doi:10.1185/03007990 6X158066

Jiang, R., Yamaori, S., Takeda, S., Yamamoto, I., \& Watanabe, K. (2011). Identification of cytochrome $\mathrm{P} 450$ enzymes responsible for metabolism of cannabidiol by human liver microsomes. Life Sciences, 89(5-6), 165-170. PubMed ID: 21704641 doi:10.1016/j.lfs.2011.05.018

Kasper, A. M., Sparks, S. A., Hooks, M., Skeer, M., Webb, B., Nia, H., . . . Close, G. L. (2020). High prevalence of cannabidiol use within male professional rugby union and league players: A quest for pain relief and enhanced recovery. International Journal of Sport Nutrition Exercise Metabolism, 30(5), 315-322. doi:10.1123/ijsnem.20200151

Lang, C. H., Frost, R. A., Deshpande, N., Kumar, V., Vary, T. C., Jefferson, L. S., \& Kimball, S. R. (2003). Alcohol impairs leucinemediated phosphorylation of 4E-BP1, S6K1, eIF4G, and mTOR in skeletal muscle. American Journal of Physiology-Endocrinology Metabolism, 285(6), E1205-E1215. PubMed ID: 12944322 doi:10. 1152/ajpendo.00177.2003

Lattanzi, S., Brigo, F., Trinka, E., Zaccara, G., Cagnetti, C., Del Giovane, C., \& Silvestrini, M. J. D. (2018). Efficacy and safety of cannabidiol in epilepsy: A systematic review and meta-analysis. Drugs, 78(17), 1791-1804. PubMed ID: 30390221 doi:10.1007/ s40265-018-0992-5

Machado Bergamaschi, M., Helena Costa Queiroz, R., Waldo Zuardi, A., \& Crippa, A. S. (2011). Safety and side effects of cannabidiol, a Cannabis sativa constituent. Current Drug Safety, 6(4), 237-249. doi:10.2174/157488611798280924

Mackey, A. L., Kjaer, M., Dandanell, S., Mikkelsen, K. H., Holm, L., Døssing, S., ... Schrøder, H. D. (2007). The influence of antiinflammatory medication on exercise-induced myogenic precursor cell responses in humans. Journal of Applied Physiology, 103(2), 425-431. PubMed ID: 17463304 doi:10.1152/japplphysiol.00157. 2007 
Mizushima, N., \& Yoshimori, T. (2007). How to interpret LC3 immunoblotting. Autophagy, 3(6), 542-545. PubMed ID: 17611390 doi:10. 4161/auto.4600

Palazzoli, F., Citti, C., Licata, M., Vilella, A., Manca, L., Zoli, M., ... Cannazza, G. (2018). Development of a simple and sensitive liquid chromatography triple quadrupole mass spectrometry (LC-MS/MS) method for the determination of cannabidiol (CBD), $\Delta 9$-tetrahydrocannabinol (THC) and its metabolites in rat whole blood after oral administration of a single high dose of CBD. Journal of Pharmaceutical Biomedical Analysis, 150, 25-32. PubMed ID: 29202305 doi:10.1016/j.jpba.2017.11.054

Parr, E. B., Camera, D. M., Areta, J. L., Burke, L. M., Phillips, S. M., Hawley, J. A., \& Coffey, V. G. (2014). Alcohol ingestion impairs maximal post-exercise rates of myofibrillar protein synthesis following a single bout of concurrent training. PLoS One, 9(2), e88384. PubMed ID: 24533082 doi:10.1371/journal.pone.0088384

Patra, P. H., Barker-Haliski, M., White, H. S., Whalley, B. J., Glyn, S., Sandhu, H., ... McNeish, A. J. (2019). Cannabidiol reduces seizures and associated behavioral comorbidities in a range of animal seizure and epilepsy models. Epilepsia, 60(2), 303-314. PubMed ID: 30588604 doi:10.1111/epi.14629

Pirmohamed, M., James, S., Meakin, S., Green, C., Scott, A. K., Walley, T. J., ... Breckenridge, A. M. (2004). Adverse drug reactions as cause of admission to hospital: Prospective analysis of 18820 patients. BMJ, 329(7456), 15-19. PubMed ID: 15231615 doi:10. 1136/bmj.329.7456.15

Rodemann, H., \& Goldberg, A. (1982). Arachidonic acid, prostaglandin E2 and F2 alpha influence rates of protein turnover in skeletal and cardiac muscle. Journal of Biological Chemistry, 257(4), 1632-1638. doi:10.1016/S0021-9258(19)68084-1
Schoenfeld, B. J. (2012). The use of nonsteroidal anti-inflammatory drugs for exercise-induced muscle damage. Sports Medicine, 42(12), 1017 1028. PubMed ID: 23013520 doi:10.1007/BF03262309

Soltow, Q. A., Betters, J. L., Sellman, J. E., Lira, V. A., Long, J. H., \& Criswell, D. S. (2006). Ibuprofen inhibits skeletal muscle hypertrophy in rats. Medicine Science in Sports Exercise, 38(5), 840. PubMed ID: 16672835 doi:10.1249/01.mss.0000218142.98704.66

Trappe, T. A., White, F., Lambert, C. P., Cesar, D., Hellerstein, M., Evans, W. J., \& Metabolism. (2002). Effect of ibuprofen and acetaminophen on postexercise muscle protein synthesis. American Journal of Physiology-Endocrinology And Metabolism, 282(3), E551-E556.

U.S. Food and Drug Administration. (2020). FDA regulation of cannabis and cannabis-derived products, including cannabidiol (CBD). Retrieved from https://www.fda.gov/news-events/public-health-focus/ fda-regulation-cannabis-and-cannabis-derived-products-includingcannabidiol-cbd

Watanabe, K., Itokawa, Y., Yamaori, S., Funahashi, T., Kimura, T., Kaji, T., ... Yamamoto, I. (2007). Conversion of cannabidiol to $\Delta 9$ tetrahydrocannabinol and related cannabinoids in artificial gastric juice, and their pharmacological effects in mice. Forensic Toxicology, 25(1), 16-21. doi:10.1007/s11419-007-0021-y

West, D. W. D., Baehr, L. M., Marcotte, G. R., Chason, C. M., Tolento, L., Gomes, A. V., .. . Baar, K. (2016). Acute resistance exercise activates rapamycin-sensitive and-insensitive mechanisms that control translational activity and capacity in skeletal muscle. The Journal of Physiology, 594(2), 453-468. PubMed ID: 26548696 doi:10.1113/JP271365

Zeiger, J. S., Silvers, W. S., Fleegler, E. M., \& Zeiger, R. S. (2019). Cannabis use in active athletes: Behaviors related to subjective effects. PLoS One, 14(6), e0218998. PubMed ID: 31251769 doi:10. 1371/journal.pone.0218998 